TITLE:

\title{
New process for catalyst-free biodiesel production using subcritical acetic acid and supercritical methanol
}

\section{AUTHOR(S):}

Saka, Shiro; Isayama, Yohei; Ilham, Zul; Jiayu, Xin

\section{CITATION:}

Saka, Shiro ...[et al]. New process for catalyst-free biodiesel production using subcritical acetic acid and supercritical methanol. Fuel 2010, 89(7): 1442-1446

ISSUE DATE:

2010-07

URL:

http://hdl.handle.net/2433/120333

\section{RIGHT:}

(c) 2010 Elsevier B.V.; この論文は出版社版でありません。引用の際には 出版社版をご確認ご利用ください。; This is not the published version. Please cite only the published version. 


\title{
New Process for Catalyst-Free Biodiesel Production using Subcritical Acetic Acid and Supercritical Methanol
}

\author{
Shiro Saka*, Yohei Isayama, Zul Ilham and Xin Jiayu \\ Department of Socio-Environmental Energy Science, Graduate School of Energy Science, Kyoto-University, \\ Yoshida-honmachi, Sakyo-ku, Kyoto 606-8501, Japan
}

\begin{abstract}
The production of glycerol as a by-product is unavoidable in the current conventional biodiesel manufacturing processes. Since biodiesel production is expected to increase in the near future, effective utilization of glycerol will become an issue of interest. In this study, therefore, a process consisting of subcritical acetic acid treatment to convert rapeseed oil to fatty acids and triacetin followed by conversion of the obtained fatty acids to their fatty acid methyl esters in supercritical methanol treatment was investigated. The obtained results clearly revealed that this two-step reaction could proceed effectively at a high reaction rate, and that fatty acid methyl esters and triacetin could be obtained under milder reaction condition than the one-step process utilizing supercritical methyl acetate and supercritical methanol.
\end{abstract}

Keywords: Biodiesel; Supercritical process; Acetic acid; Triacetin; Catalyst-free process 


\section{Introduction}

Biodiesel fuel (BDF) derived from vegetable oils and their wastes has recently received a lot of attentions as an alternative to fossil fuel. In the current commercial practice of BDF production by alkali-catalyzed method, triglycerides (TG) are transesterified to fatty acid methyl esters (FAME) with methanol in the presence of an alkaline catalyst. Therefore, what is generally referred to as BDF is a mixture of various FAME.

Although alkali-catalyzed method has the benefit of proceeding in a moderate reaction condition, several aqueous washing-cycles are required to remove the catalyst and other impurities after the reaction. However, water and free fatty acids are contained in the oils and fats. The former contributes to a reduction of the catalytic effect, while the latter could react with the catalyst to produce alkaline soaps, leading to a reduction in the yield of FAME. Therefore, manufacturing BDF from waste cooking oils with water and free fatty acids by the alkali-catalyzed method is complicated [1, 2].

Non-catalytic BDF production methods using supercritical methanol have been developed to resolve various problems in the above-mentioned techniques [3]. In the one-step supercritical methanol method (Saka Method), the transesterification reaction of triglycerides from oils or fats proceeds without any catalysts in supercritical methanol. In addition, raw material of oils or fats with high content of free fatty acids could also be converted to FAME simultaneously by the esterification reaction in supercritical methanol [4]. From this process, a high yield of FAME could be obtained, and there are no alkaline soaps generated . Moreover, separation and purification of the products are easy. Unlike the alkali-catalyzed 
method, this method could also be applied to relatively long chain alcohols [5]. However, this method requires extreme reaction conditions of $350^{\circ} \mathrm{C}$ and $43 \mathrm{MPa}$, where the isomerization of unsaturated fatty acids from the cis-type to the trans-type and the decomposition of unsaturated fatty acids could be induced, resulting in adverse effects on the cold flow properties of the fuel [6, 7].

For this reason, Kusdiana and Saka developed the two-step supercritical methanol method (Saka-Dadan Method) for BDF production, which proceeds in a milder reaction conditions [8]. In this method, the hydrolysis reaction of triglycerides is induced in subcritical water to fatty acids and glycerol. It was, then, followed by a subsequent esterification of the obtained fatty acids in supercritical methanol into FAME. Since the reaction condition in this method is relatively mild at $270^{\circ} \mathrm{C}$ and $7 \mathrm{MPa}$, the possibility of unsaturated fatty acids isomerization to trans-type and thermal decomposition of unsaturated fatty acids could be avoided [7]. Some other researchers later discuss further in this area by incorporating analysis of energy consumption on industrial scale [9] and assessment for new or additional research for advances in this field [10].

However, in these methods, the production of glycerol as a by-product could not be avoided. Therefore, with increased production of BDF in years to come, the production of glycerol is also expected to increase precipitously. However, in alkali-catalyzed method, glycerol phase contains methanol, water, alkaline catalyst and soap, thus, the sale value of this crude glycerol is extremely low at approximately $\$ 0.1 / \mathrm{kg}$, compared with the purified glycerol at approximately $\$ 1.3 \sim 2.0 / \mathrm{kg}$. When the cost of transportation is taken into consideration, the market price is, in fact, uneconomical [11]. Therefore, an effective method 
for BDF production without the production of glycerol is worth to be established.

To deal with this, Fabbri et al. have proposed an alkali-catalyzed transesterification of oils and with dimethyl carbonate to derive FAME and cyclic glycerol carbonate esters of fatty acids (FAGC) [12]. Ilham and Saka also studied the potential of dimethyl carbonate as a reactant in non-catalytic supercritical process. This method is a promising process to produce higher value by-products than glycerol [13]. However, since the former process by Fabbri et al. utilizes alkaline catalyst, it is difficult to be applied to waste oils and fats.

In contrast, in the proposed supercritical methyl acetate method (Saka and Isayama Method), oils and fats are converted without producing glycerol to FAME and triacetin, and their mixture is used as BDF at the theoretical yield of $125 \mathrm{wt} \%$ [14]. It is further expected that BDF can be produced from all types of oils and fats. However, as the reaction condition at $350^{\circ} \mathrm{C}$ and $20 \mathrm{MPa}$ is severe, there are concerns about their effects on fuel characteristics. Therefore, in this research, the two-step process of oils and fats to fatty acids and triacetin by subcritical acetic acid followed by the fatty acid conversion to FAME by supercritical methanol was studied to produce BDF under milder reaction conditions.

\section{Materials and methods}

\subsection{Materials}

Rapeseed oil (Nacalai Tesque Inc.) was used for BDF production, while acetic acid (Nacalai Tesque Inc., 99.7\%) was used as the reaction solvent $\left(\mathrm{Tc}=317^{\circ} \mathrm{C}\right.$; Pc $=5.80 \mathrm{MPa}$ [15]) for the transesterification reaction. Moreover, triolein and methyl oleate were used as the standards for 
triglycerides and FAME, respectively (both manufactured by Sigma-Aldrich, $\geq 99 \%$ ), while triacetin as that for the final product (manufactured by Nacalai Tesque Inc., 98\%). Distilled water was used to wash the reactants after the transesterification reaction, and methanol $\left(\mathrm{Tc}=239^{\circ} \mathrm{C} ; \mathrm{Pc}=8.09 \mathrm{MPa}[16]\right)$ was used as the solvent in the esterification reaction (both being HPLC grade, manufactured by Nacalai Tesque Inc.).

To obtain the authentic standards for monoacetyl diglyceride and diacetyl monoglyceride, excess of acetic anhydride (manufactured by Nacalai Tesque Inc., 97\%) and pyridine (manufactured by Nacalai Tesque Inc., 99.5\%) were added to 1,3-diolein and 1-oleyl-rac-glycerol (both manufactured by Sigma-Aldrich, 99.5\%), respectively, and reacted overnight.

\subsection{Experimental procedures}

For subcritical acetic acid treatment of rapeseed oil, a flow-type supercritical fluid conversion system was used. Rapeseed oil and solvents were fed by high pressure pump from the raw material and solvent tanks, respectively. After mixing these in the reaction tube orifice, they were led into the reaction tube in a molten salt bath (volume: $288 \mathrm{~mL}$, material: Hastelloy C276) maintaining at a specific temperature to perform the supercritical treatment. The reaction time was controlled by the flow volume setting of the high-pressure pump, while the pressure was controlled by adjusting the back-pressure regulator. After the processing in the reaction vessel, the reaction was stopped by using a cooler and the product was recovered.

The reaction condition for the subcritical and supercritical acetic acid processing of the oil was at temperatures of $250-320^{\circ} \mathrm{C}$ under $20 \mathrm{MPa}$, while the reaction time was $5-40 \mathrm{~min}$. Moreover, the molar ratio 
of the oil to acetic acid was 1:54 (1:3.2 by volumetric ratio).

After the subcritical and supercritical treatment, the remained acetic acid were removed by distillation in a rotary evaporator. Then, $20 \mathrm{ml}$ distilled water was added to $5 \mathrm{~g}$ reactants and stirred for a while, and the oil and water layers were separated in a centrifugal separator. Only the oil layer was supplied for the subsequent supercritical methanol process.

A batch-type supercritical fluid conversion system was used for the supercritical methanol treatment of the oil layer at temperatures of $270-300^{\circ} \mathrm{C}$ and pressures of $17-20 \mathrm{MPa}$ for $2-30 \mathrm{~min}$. The volumetric ratio of reactants to methanol was 1:1.6 (molar ratio was 1:14 if reactants were fatty acids). Thereafter, the FAME was obtained by removal of the methanol in a rotary evaporator [14].

\subsection{Analysis}

The reactants derived from subcritical and supercritical treatments of rapeseed oil with acetic acid were analyzed by gel permeation chromatography (GPC) (column: GF-310HQ, oven temperature: $40^{\circ} \mathrm{C}$, flow-rate: $1.0 \mathrm{~mL} / \mathrm{min}$, mobile phase: tetrahydrofuran (THF), detector: refractive index detector (RID)). Moreover, the oil layer obtained by aqueous washing of the reactants after subcritical processing was analyzed by GPC in the same process, and the aqueous layer was analyzed by a high performance liquid chromatography (HPLC) (column: ULTRON PS-80P, oven temperature: $40^{\circ} \mathrm{C}$, flow-rate: $1.0 \mathrm{~mL} / \mathrm{min}$, mobile phase: distilled water, detector: refractive index detector (RID)). Furthermore, the reactants derived from supercritical methanol treatment of oil were also analyzed by GPC (column: GF-310HQ, oven 
temperature: $40^{\circ} \mathrm{C}$, flow-rate: $1.0 \mathrm{~mL} / \mathrm{min}$, mobile phase: acetone, detector: RID). For the calibration of the curve of the standard products, triolein, 1,3-dioleyl-2-acetyl-glycerol (monoacetyl diglyceride), 1-oleyl-2,3-diacetyl-glycerol (diacetyl monoglyceride), methyl oleate, and triacetin were used.

The yields of fatty acid $\mathrm{y}_{\mathrm{FA}}$ and triacetin $\mathrm{y}_{\mathrm{TAa}}$ obtained by the subcritical and supercritical acetic acid treatments of rapeseed oil were calculated as the theoretical maximum concentration value of $100 \mathrm{wt} \%$, based on the area of the GPC chromatographic peak. Furthermore, the triacetin yield $\mathrm{y}_{\mathrm{TAw}}$ from the water layer after aqueous wash was also calculated as the theoretical maximum concentration value (where the transesterification reaction had proceeded completely, and all triacetin produced was recovered from the water layer) of 100wt\%, based on the area of the GPC chromatographic peak.

With respect to the yields of FAME $\mathrm{y}_{\mathrm{FAME}}$, after supercritical methanol treatment, although the composition of materials actually supplied for treatment was complex, rapeseed oil before treatment was assumed to contain $100 \mathrm{wt} \%$ triglycerides. Moreover, with a view to use the FAME and triacetin together as BDF after supercritical methanol treatment of the obtained fatty acids, BDF yield $\left(\mathrm{Y}_{\mathrm{BDF}}\right)$ was defined as the total amount of FAME and triacetin generated per unit of triglycerides. Therefore, in this method, the maximum theoretical value for BDF yield is $125 \mathrm{wt} \%$ [14].

\section{Results and Discussion}

\subsection{Transesterification of triglycerides with acetic acid}

The yield of fatty acids as a result of rapeseed oil treatment with subcritical and supercritical 
acetic acid $\left(\mathrm{Tc}=317^{\circ} \mathrm{C}\right.$; Pc $\left.=5.80 \mathrm{MPa}\right)$ is shown in Fig. 1 , and the triacetin yield is shown in Fig. 2. Fatty acids and triacetin were generated from the oil and acetic acid at temperatures ranging from 250 to $320^{\circ} \mathrm{C}$ at $20 \mathrm{MPa}$ in pressure, and it is clear that the transesterification reaction shown in reaction (1) proceeds. Therefore, it is apparent that the reaction could proceed without the addition of a catalyst in a transesterification of triglycerides with acetic acid under subcritical and supercritical conditions.

In subcritical processing at $300^{\circ} \mathrm{C} / 20 \mathrm{MPa}$, a yield of $91 \mathrm{wt} \%$ of fatty acid was obtained after 30 min treatment, and remained at a constant value even after prolonged reaction time. In contrast, at the supercritical condition of $320^{\circ} \mathrm{C} / 20 \mathrm{MPa}$, approximately $88 \mathrm{wt} \%$ fatty acid yield was obtained after 5 min reaction time, and the maximum yield was $92 \mathrm{wt} \%$. Moreover, treatments at relatively low reaction temperatures of $250^{\circ} \mathrm{C}$ and $270^{\circ} \mathrm{C}$, could also generate fatty acids of 39 and $73 \mathrm{wt} \%$ after 40 min treatment, respectively.

In contrast, the generation of triacetin was hardly observed even after 40 min of treatment at $250^{\circ} \mathrm{C}$, and the generated amount was somewhat less than that of the fatty acids. However, when the reaction temperature was increased to above $290^{\circ} \mathrm{C}$, the amount of triacetin generated was increased, and showed almost the same trend as seen in the fatty acid yield. The maximum yield of triacetin was observed at $300^{\circ} \mathrm{C}$ after $30 \mathrm{~min}$ treatment, and the obtained yield was $85 \mathrm{wt} \%$, which is slightly lower, compared with the fatty acid yield. 


\subsection{Kinetics on transesterification of triglycerides with acetic acid}

The overall reaction for the first step, transesterification of triglycerides with acetic acid is shown below:

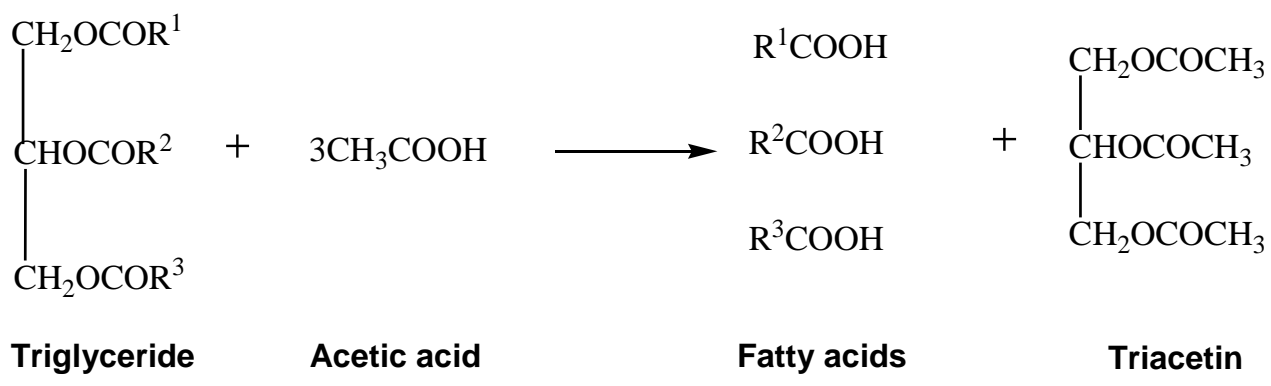

In fact, the above-mentioned reaction is expected to proceed in three stages. First, triglyceride

and acetic acid react, one molecule at a time, to generate one molecule each of monoacetyl diglyceride and fatty acid. Similarly, diacetyl monoglyceride and fatty acid are generated from monoacetyl diglyceride and acetic acid, and triacetin and fatty acid are generated from diacetyl monoglyceride and acetic acid.

In this study, due to the possibility of some reverse reactions to occur, six reaction rate constants should be used for a strict theoretical reaction rate analysis. However, in this study, because excessive acetic acid with respect to triglyceride was added, it was assumed that the reaction proceeded as pseudo-first-order, and to simplify the performance of analysis in the modeling, Eq. (2) was used.

$$
\frac{d[T G]}{d t}=-k_{I E}[T G]
$$

where [TG] is the molar concentration (mol\%) of triglyceride and $\mathrm{k}_{\mathrm{IE}}$ is the rate constant $\left(\mathrm{s}^{-1}\right)$.

In Eq. (3), derived from Eq. (2), $\mathrm{k}_{\mathrm{IE}}$ is evaluated by deriving the regression curve. This curve is derived by the Levenberg-Marquardt method.

$$
[T G]=e^{-k_{I E} t}[T G]_{0}
$$


The Arrhenius plot of the rate constant for the transesterification of rapeseed oil in subcritical and supercritical processing is shown in Fig. 3. For comparison, the results of the transesterification reactions using methanol and methyl acetate are shown as dotted lines [14, 17]. It can be seen that the reactivity of acetic acid in the transesterification reaction is higher than those of both methanol and methyl acetate.

The higher reactivity of acetic acid in the transesterification reaction is believed to be due to the excessive amount of acetic acid used. In other words, although acetic acid is one of the reactant, for the transesterification, it is believed that it also acts as an acid catalyst. Moreover, although acetic acid is consumed as the reaction proceeds, it is replaced by the same molar ratio of generated fatty acids, and these are believed to have the same acid catalytic effect [18]. Therefore, in this first step, the reactions proceeded under conditions of high acid catalyst concentration. For these reasons, as acetic acid was used, the reaction rate was higher, compared with methanol or methyl acetate used.

For the second step which is the treatment of fatty acids in supercritical methanol, extensive kinetic works have been studied by Minami and Saka [18]. The reaction was consequently found to proceed by the autocatalytic mechanism of fatty acids itself in supercritical methanol.

\subsection{Yield of fatty acid methyl esters}

Figure 4 shows the FAME yield as treated in subcritical and supercritical acetic acid followed derived by supercritical methanol treatments at two reaction conditions of $300^{\circ} \mathrm{C} / 20 \mathrm{MPa}$ and $270^{\circ} \mathrm{C} / 17 \mathrm{MPa}$. As a result, the esterification reaction reached an equilibrium state after $2 \mathrm{~min}$ at $300^{\circ} \mathrm{C}$ and $15 \mathrm{~min}$ at $270^{\circ} \mathrm{C}$ 
with a 95-96 wt\% yield of FAME. Because this yield is higher than that of fatty acids obtained after subcritical acetic acid treatment, it is believed that reaction intermediates such as diacetyl monoglyceride and monoacetyl diglyceride, reacted with methanol into FAME.

The results in this study are significant enough when compared with previous results by other researchers. Du et al. in their study with methyl acetate as acyl acceptor, achieved 92\% yield of FAME after 14h of reaction [19], while Modi et al. obtained the maximum yield up to 93\% with ethyl acetate after $12 \mathrm{~h}$ reaction [20]. Proved by these comparisons, the non-catalytic two-step process presented in this study proves to be highly potential as a milder biodiesel production in subcritical and supercritical condition.

\subsection{BDF production using subcritical acetic acid and supercritical methanol}

The BDF production process comprising of subcritical acetic acid processing and supercritical methanol processing is shown in Fig. 5. In this process, the raw material (oil and fat) is subcritically treated with acetic acid to obtain fatty acids and triacetin. After removal of the acetic acid from the reactants, the triacetin was recovered by several aqueous washes, and the fatty acids were, then, supplied to the supercritical methanol treatment. In addition to the FAME being produced from the fatty acids, FAME is also produced from unreacted triglyceride and reaction intermediates such as diacetyl monoglyceride or monoacetyl diglycerides. Thereafter, the obtained FAME and triacetin are used together as BDF. The subcritical acetic acid treatment condition for rapeseed oil was $300^{\circ} \mathrm{C} / 20 \mathrm{MPa}$ for $30 \mathrm{~min}$ at a molar ratio of 1:54 (volumetric ratio of 1:3.2), whereas the supercritical methanol treatment conditions for fatty acids was 
$270^{\circ} \mathrm{C} / 17 \mathrm{MPa}$ for $15 \mathrm{~min}$ at a molar ratio of $1: 14$ (volumetric ratio of $1: 1.6$ ). Where triacetin was

completely recovered as BDF, the yield of FAME was $97 \mathrm{wt} \%$ and that of triacetin was $20 \mathrm{wt} \%$, totally being $117 \mathrm{wt} \%$ for the maximum theoretical value of $125 \mathrm{wt} \%$. Moreover, in this process, as the triacetin was generated unintentionally, it is conceivable that it may be recovered and used effectively as a useful chemical.

\section{Concluding Remarks}

This study was aimed at solving the problem of waste glycerol in the current BDF production processes by investigating a BDF production process by combination of the subcritical treatment with acetic acid and subsequent supercritical treatment with methanol. As a result, a transesterification reaction was preceded between acetic acid and triglyceride under subcritical condition without the addition of catalysts, and it became clear that fatty acids and triacetin could be obtained in a high yield. When acetic acid was used, the reaction rate was much higher than when either of methyl acetate or methanol in the transesterification. In addition, the recovery of triacetin from subcritical acetic acid treatment by aqueous washing was determined at $64 \%$. On the other hand, when the oil phase containing fatty acids from the previous treatment was supplied for the supercritical methanol process at $270^{\circ} \mathrm{C} / 17 \mathrm{MPa}$ for $15 \mathrm{~min}$, a 97 wt\% yield of FAME was achieved.

The recovery rate of BDF from this process was $117 \mathrm{wt} \%$, a value which is higher than that obtained by the conventional BDF manufacturing process, which produces glycerol as a by-product. Its 
recovery rate is also higher than the recently reported supercritical methyl acetate method (Saka and Isayama Method). In both subcritical acetic acid process and supercritical methanol process, a good reactivity was found at a relatively low temperature, suggesting the possibility that energy efficiency could be further increased by reducing the molar ratio of reaction solvent. Although future investigations on the BDF fuel characteristics of the obtained mixture of FAME and triacetin are needed, this study has already revealed a promising superior BDF production method that does not produce glycerol as a by-product.

\section{Acknowledgments}

This study is a part of NEDO "High Efficiency Bioenergy Conversion Projects" in FY2007-08, for which the authors are highly acknowledged.

\section{References}

[1] Mittelbach M, Remschmidt C. Oil-pretreatment. In: Biodiesel. Vienna: Boersedruck Ges.m.b.H; 2004, p.44-46.

[2] Tomasevic AV, Marinkovic SSS. Methanolysis of used frying oil. Fuel Proc Technol 2003;81:1-6.

[3] Saka S, Kusdiana D. Biodiesel fuel from rapeseed oil as prepared in supercritical methanol. Fuel 2001;80:225-231.

[4] Kusdiana D, Saka S. Methyl esterification of free fatty acids of rapeseed oil as treated in supercritical methanol. J Chem Eng. Jpn 2001;34:383-387. 
[5] Warabi Y, Kusdiana D, Saka S. Biodiesel fuel from vegetable oil by various supercritical alcohols. Appl Biochem Biotechnol 2004;115:793-801.

[6] Tabe A, Kusdiana D, Minami E, Saka S. Kinetics in transesterification of rapeseed oil by supercritical methanol treatment. In; Proc of the 2nd World Biomass Conf - Biomass for Energy, Ind and Climate Prot 2004, p.1553-1556.

[7] Imahara H, Minami E, Hari S, Saka S. Thermal stability of biodiesel in supercritical methanol. Fuel 2008;87:1-6.

[8] Kusdiana D, Saka S. Two-step preparation for catalyst-free biodiesel fuel production. Appl Biochem Biotechnol 2004;115:781-791.

[9] Glisic D, Lukic I, Skala D. Biodiesel synthesis at high pressure and temperature: Analysis for energy consumption on industrial scale. Bioresour Technol 2009;24:6347-6354.

[10] Pinnarat T, Savage PE. Assessment of non-catalytic biodiesel synthesis using supercritical reaction conditions. Ind Eng Chem Res 2008;47:6801-6808.

[11] Johnson DT, Taconi KA. The glycerin glut: Options for the value-added conversion of crude glycerol resulting from biodiesel production. Environ Progress 2007;26:338-348.

[12] Fabbri D, Bevoni V, Natari M, Rivetti F. Properties of a potential biofuel obtained from soybean oil by transesterification with dimethyl carbonate. Fuel 2007;86:690-697.

[13] Ilham Z, Saka S. Dimethyl carbonate as potential reactant in non-catalytic biodiesel production by supercritical method. Bioresour Technol 2009;100:1793-1796. 
[14] Saka S, Isayama Y. New process for catalyst-free biodiesel production with supercritical methyl acetate. Fuel 2009;88:1307-1313.

[15] Nikitin ED, Pavlov PA, Popov AP. Critical temperatures and pressures of some alkanoic acids (C2 to C22) using the pulse-heating method. Fluid Phase Equilibria 2001;189(1-2):151-161.

[16] Goodwin RD. Methanol thermodynamic properties from 176 to $673 \mathrm{~K}$ at pressures to 700 bar. J Phys Chem Ref Data 1987;16(4):799-892.

[17] Kusdiana D, Saka S. Kinetics of transesterification in rapeseed oil to biodiesel fuel as treated in supercritical methanol. Fuel 2001;80:693-698.

[18] Minami E, Saka S. Kinetics of hydrolysis and methyl esterification for biodiesel production in two-step supercritical methanol process. Fuel 2006;85:2479-2483.

[19] Du W, Xu Y, Liu D, Zeng J. Comparative study in lipase-catalyzed transformation of soybean oil for biodiesel production with different acyl acceptors. J Mol Catal 2004;30:125-129.

[20] Modi MK, Reddy JRC, Rao BVSK, Prasad RBN. Lipase-mediated conversion of vegetable oils into biodiesel using ethyl acetate as acyl acceptor. Bioresour Technol 2007;98:1260-1264. 


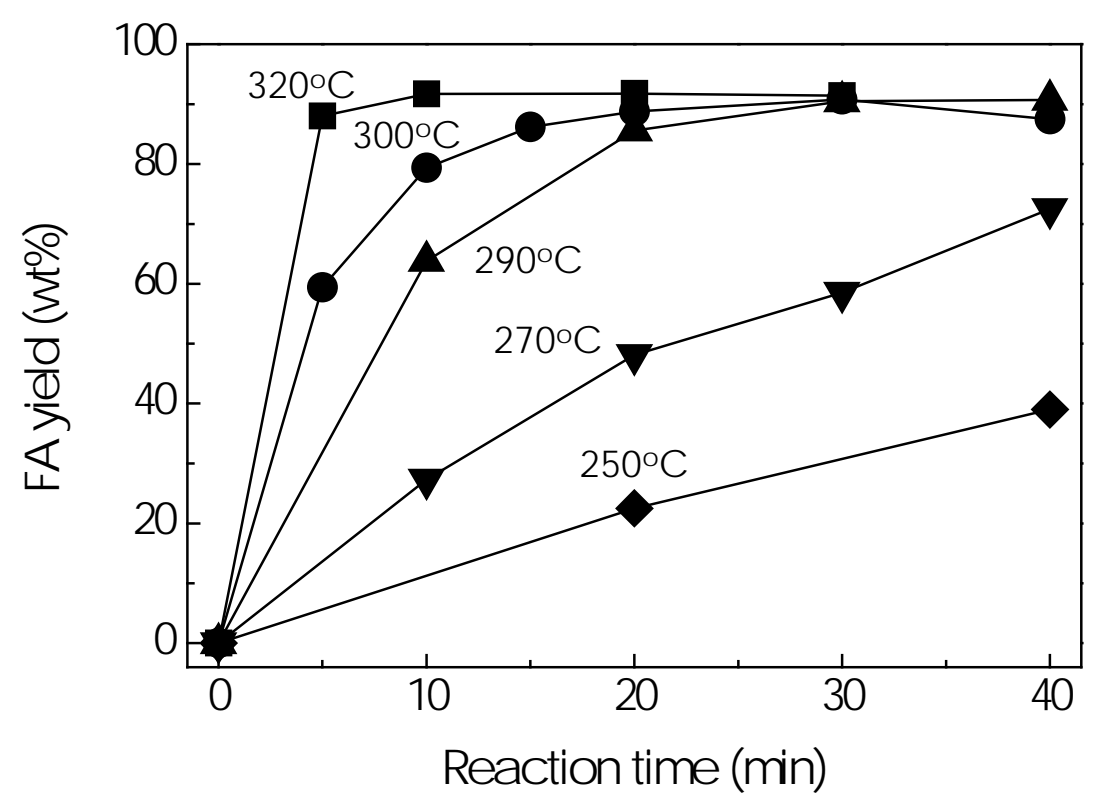

Fig. 1. Yields of fatty acids (FA) from rapeseed oil as treated in subcritical and supercritical acetic acid at various temperatures in 20MPa (acetic acid/oil = 54/1 (mol/mol)). 


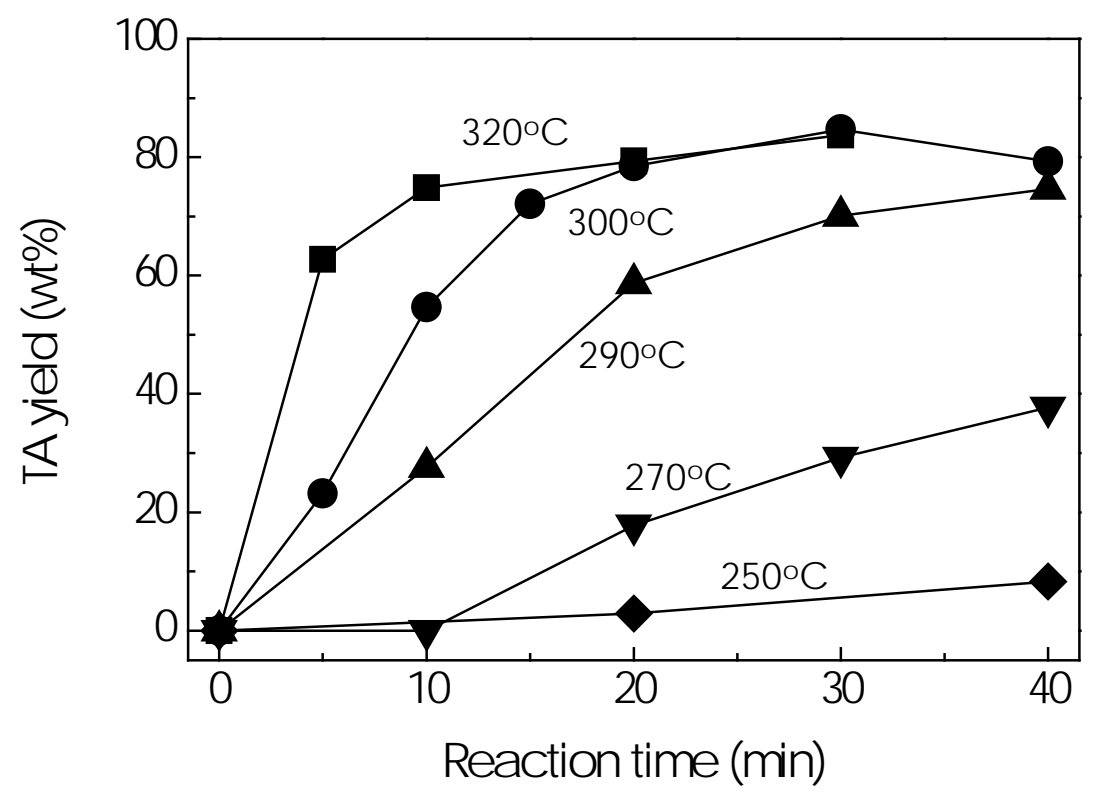

Fig. 2. Yields of triacetin (TA) from rapeseed oil as treated in subcritical and supercritical acetic acid at various temperatures in 20MPa (acetic acid/oil $=54 / 1(\mathrm{~mol} / \mathrm{mol})$ ). 


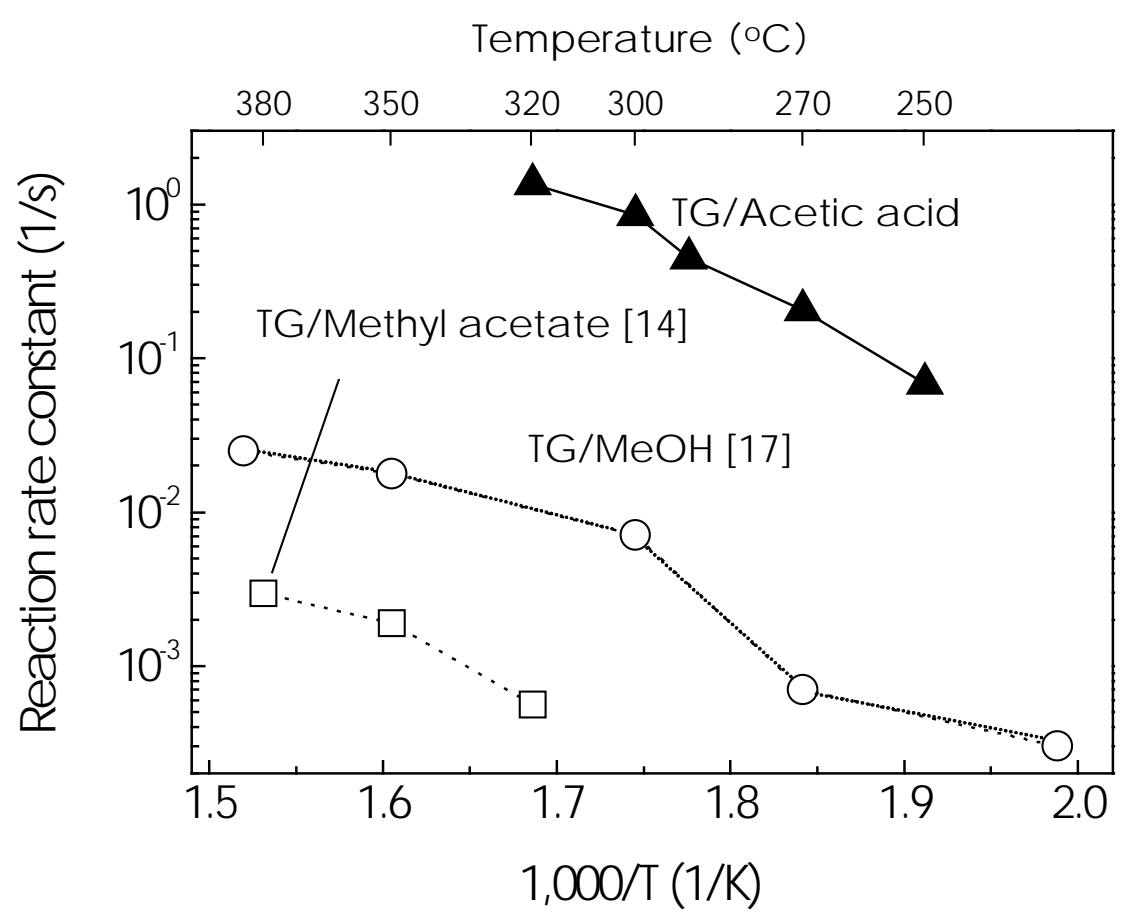

Fig. 3. Arrhenius plots for reaction rate constants on transesterification by subcritical and supercritical acetic acid at 20MPa. For comparison, the results with methanol in batch-type system and with methyl acetate in flow-type system at 20MPa are included [14,17]. 


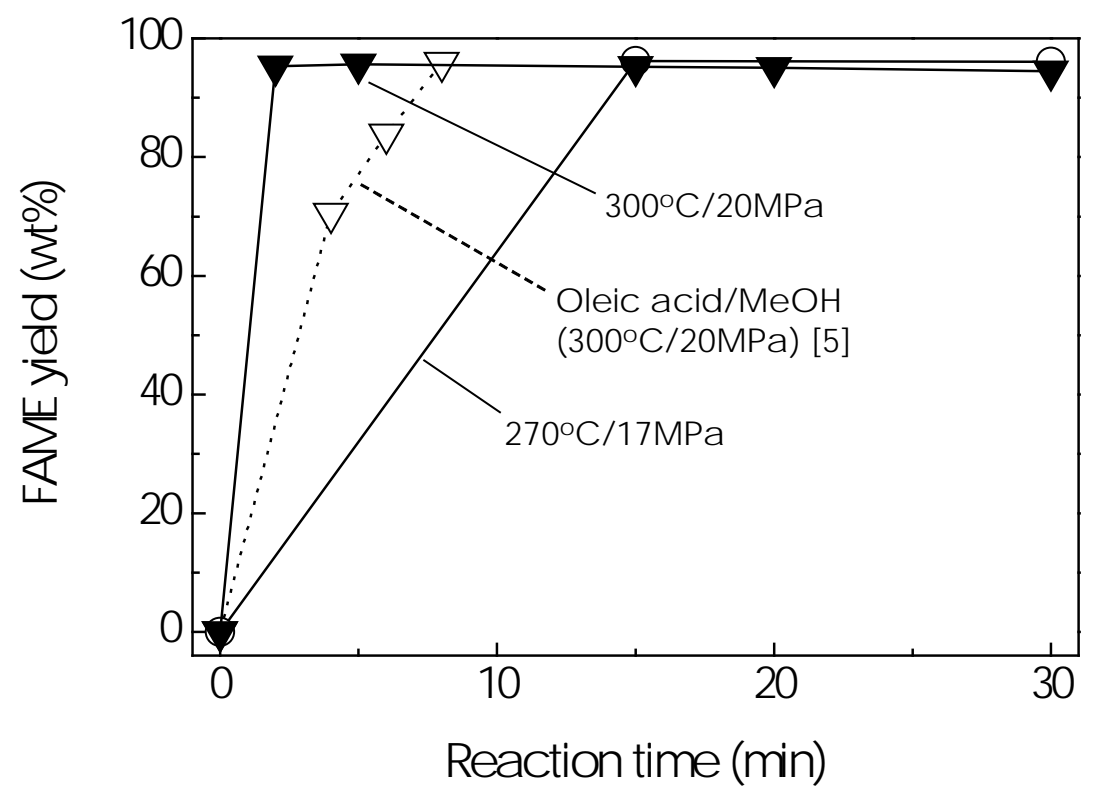

Fig. 4. Yields of fatty acid methyl esters (FAME) as treated in subcritical acetic acid at $300^{\circ} \mathrm{C} / 20 \mathrm{MPa}$ followed by supercritical methanol treatments at $300^{\circ} \mathrm{C} / 20 \mathrm{MPa}$ and $270^{\circ} \mathrm{C} / 17 \mathrm{MPa}$. For comparison, the results of oleic acid and methanol are included [5]. 


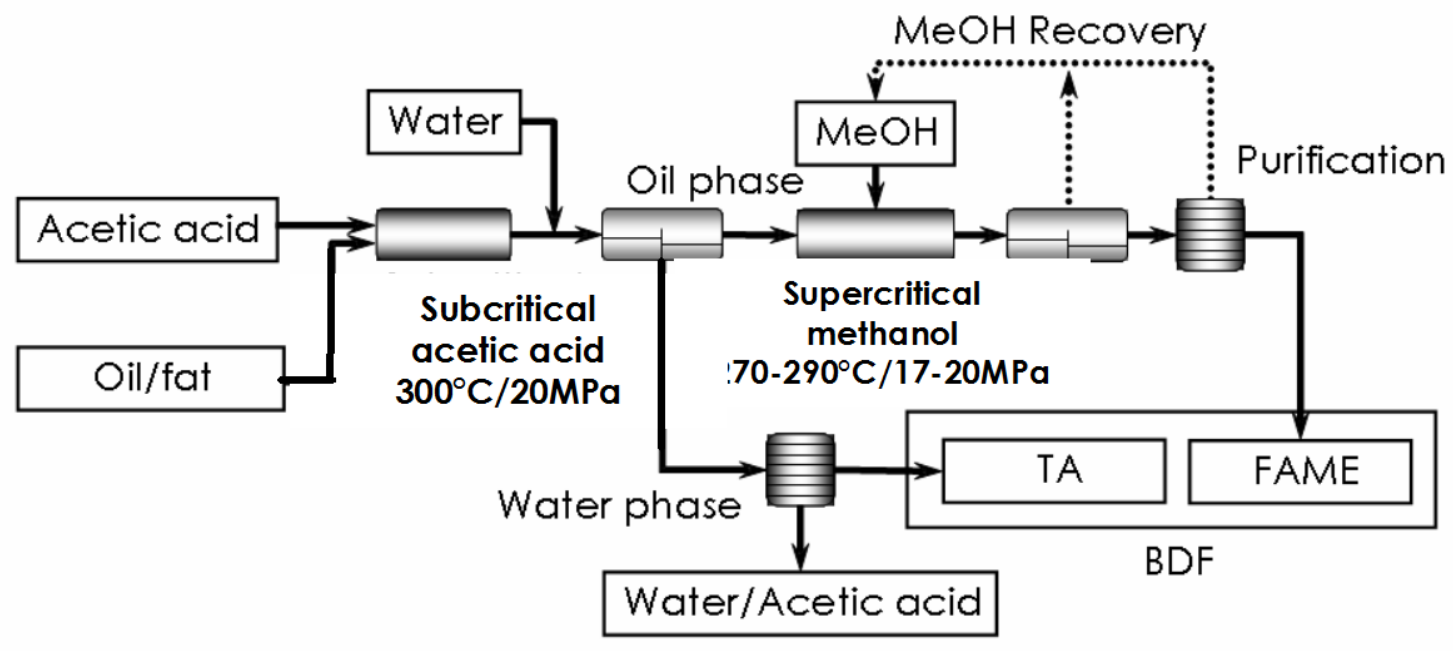

Fig. 5. Process for catalyst-free biodiesel production with subcritical acetic acid and supercritical methanol. 